\title{
Testbed demonstration of high- contrast coronagraph imaging in search for Earth-like exoplanets
}

Seo, Byoung-Joon, Patterson, Keith, Balasubramanian, Kunjithapatham, Crill, Brendan, Chui, Talso, et al.

Byoung-Joon Seo, Keith Patterson, Kunjithapatham Balasubramanian, Brendan Crill, Talso Chui, Daniel Echeverri, Brian Kern, David Marx, Dwight Moody, Camilo Mejia Prada, Garreth Ruane, Fang Shi, John Shaw, Nick Siegler, Hong Tang, John Trauger, Daniel Wilson, Robert Zimmer, "Testbed demonstration of high-contrast coronagraph imaging in search for Earth-like exoplanets," Proc. SPIE 11117, Techniques and Instrumentation for Detection of Exoplanets IX, 111171V (16 September 2019); doi: 10.1117/12.2530033

Event: SPIE Optical Engineering + Applications, 2019, San Diego, California, United States 


\title{
Testbed demonstration of high-contrast coronagraph imaging in search for Earth-like exoplanets
}

\author{
Byoung-Joon Seo ${ }^{a}$, Keith Patterson ${ }^{a}$, Kunjithapatham Balasubramanian ${ }^{a}$, Brendan Crill ${ }^{a}$, \\ Talso Chui ${ }^{a}$, Daniel Echeverri ${ }^{b}$, Brian $\mathrm{Kern}^{a}$, David $\mathrm{Marx}^{a}$, Dwight $\mathrm{Moody}^{a}$, Camilo Mejia \\ Prada $^{a}$, Garreth Ruane ${ }^{a}$, Fang Shi ${ }^{a}$, John Shaw ${ }^{a}$, Nick Siegler ${ }^{a}$, Hong Tang ${ }^{a}$, John Trauger ${ }^{a}$, \\ Daniel Wilson ${ }^{a}$ and Robert Zimmer ${ }^{a}$ \\ ${ }^{a}$ Jet Propulsion Laboratory, California Institute of Technology, 4800 Oak Grove Dr., \\ Pasadena, CA. 91109, USA \\ ${ }^{b}$ Department of Astronomy, California Institute of Technology, 1200 E. California Blvd., \\ Pasadena, CA. 91125, USA
}

\begin{abstract}
Direct imaging of an Earth-like exoplanet requires starlight suppression with a contrast ratio on the order of $1 \times 10^{-10}$ at small angular separations of 100 milliarcseconds or less in visible light with more than $50 \mathrm{~nm}$ bandwidth. To our knowledge, the technology needed to achieve the contrast and stability has not been demonstrated as of January 2019. The science requirements for near future National Aeronautics and Space Administration (NASA) missions such as James Webb Space Telescope (JWST)'s Near Infrared Camera (NIRCam) coronagraph and Wide-Field InfraRed Survey Telescope (WFIRST) Coronagraph Instrument (CGI) are at least 10 times short. To investigate and guide the technology to reach this capability, we built a high contrast coronagraph testbed at NASA's Jet Propulsion Laboratory (JPL). Titled the Decadal Survey Testbed (DST), this state-of-art testbed is based on the accumulated experience of JPL's High Constrast Imaging Testbed (HCIT) team. Currently, the DST hosts a Hybrid Lyot Coronagraph (HLC) with an unobscured, circular pupil. The DST also has two deformable mirrors and is equipped with the Low Order Wavefront Sensing and Control (LOWFS/C) subsystem to sense and correct the dynamic wavefront disturbances. In this paper, we present up-to-date progress of the testbed demonstration. As of January 2019, we repeatedly obtain convergence below $4 \times 10^{-10}$ mean contrast with $10 \%$ broadband light centered at $550 \mathrm{~nm}$ in a 360 degrees dark hole with a working angle between 3 $\lambda / D$ and $8 \lambda / D$. We show the key elements used in the testbed and the performance results with associated analysis.
\end{abstract}

Keywords: instrumentation, exoplanets, direct detection, coronagraphs

*Address all correspondance to Byoung-Joon Seo, Email: Byoung-Joon.Seo@jpl.nasa.gov

\section{INTRODUCTION}

The goals of National Aeronautics and Space Administration (NASA)'s next generation space coronagraph missions are aligned with search for habitable Earth-like exoplanets and their direct imaging. Achieving this goal requires not only a large aperture telescope such as Large UV/Optical/IR Surveyor (LUVOIR) and Habitable Exoplanet Observatory (HabEx), ${ }^{1,2}$ but also a high performance coronagraph instrument.

Direct imaging of an Earth-like exoplanet requires starlight suppression with a contrast ratio on the order of $1 \times 10^{-10}$ at small angular separations of 100 milliarcseconds or less in visible light with more than $50 \mathrm{~nm}$ bandwidth. Such high coronagraph performance can be obtained by several key technological improvement including stable Deformable Mirror (DM), optimized coronagraph design, sensitive detectors, mechanical and thermal stability, and advanced wavefront control algorithm. To our knowledge, the technology needed to achieve the contrast and stability has not been demonstrated as of January 2019. The science requirements for near future National Aeronautics and Space Administration (NASA) missions such as James Webb Space Telescope (JWST)'s Near Infrared Camera (NIRCam) coronagraph and Wide-Field InfraRed Survey Telescope (WFIRST) Coronagraph Instrument (CGI) are at least 10 times short. $^{3}$

(C)2019 California Institute of Technology. Government sponsorship acknowledged.

Techniques and Instrumentation for Detection of Exoplanets IX, edited by Stuart B. Shaklan, Proc. of SPIE Vol. 11117, 111171V · C 2019 SPIE · CCC code: 0277-786X/19/\$21 · doi: 10.1117/12.2530033 


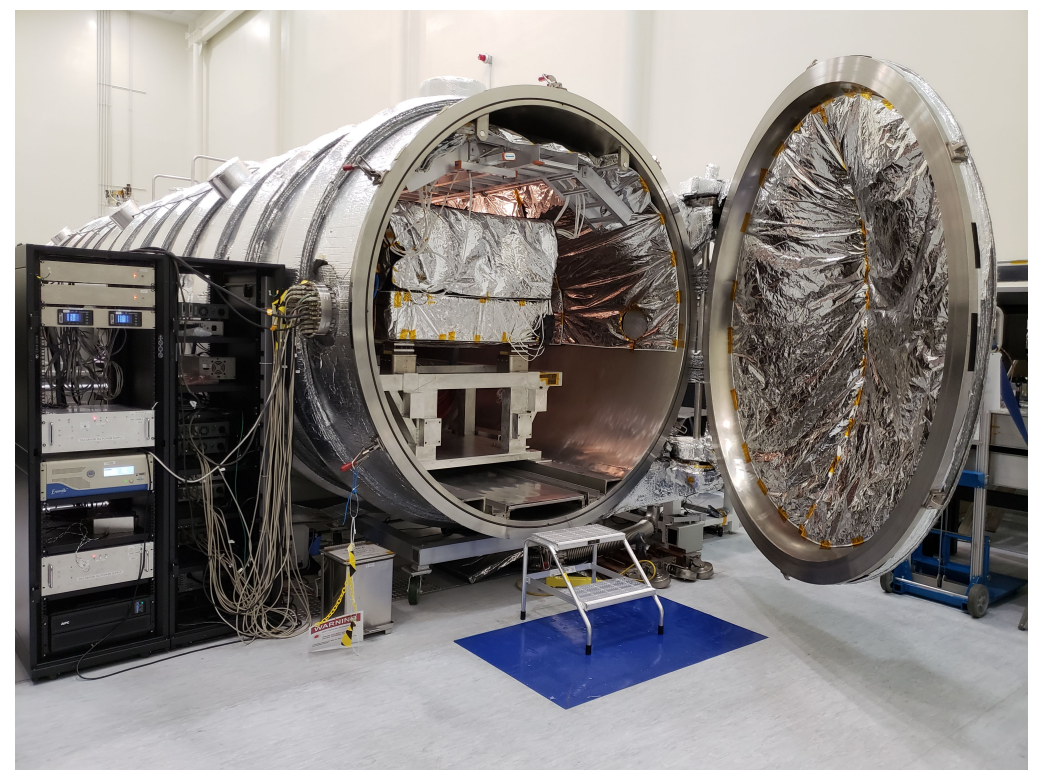

Figure 1. Photo of the DST when it is inside the vacuum chamber in JPL's HCIT.

To investigate and guide the technology to reach this capability, we, NASA's Exoplanet Exploration Program (ExEP) office, built a high contrast coronagraph testbed at NASA's Jet Propulsion Laboratory (JPL). Titled the Decadal Survey Testbed (DST), this state-of-art testbed is based on the accumulated experience of JPL's High Constrast Imaging Testbed (HCIT) team. As of January 2019, the DST is configured as its early stage (Phase A) hosting a Lyot coronagraph as its coronagraph architecture with an unobscured, circular pupil. The DST also has two DMs and is equipped with the Low Order Wavefront Sensing and Control (LOWFS/C) subsystem to sense and correct the dynamic wavefront disturbances.

We present up-to-date progress of the testbed demonstration in this paper. We repeatedly obtain convergence below $4 \times 10^{-10}$ mean contrast with $10 \%$ broadband light centered at $550 \mathrm{~nm}$ in a 360 degrees dark hole with a working angle between $3 \lambda / D$ and $8 \lambda / D$.

The remainder of this paper is structured as follows. Sec. 2 includes very brief overview of DST and refers its detail to our companion paper. ${ }^{4}$ Sec. 3 demonstrates the raw high contrast we have achieved in DST. Then, we discuss the breakdown of the contrast in Sec. 4 by showing itemized contrasts with necessary experiments and analysis. Future space telescopes with high contrast imaging instruments make use of a post processing technique to maximize visibility of the exoplanets. In Sec. 5, we apply a simple post processing algorithm, Angular Differential Imaging (ADI) technique, to the data measured from the testbed. Then, we estimate the practical planet identification capability. Finally, we conclude in Sec. 6 listing our future works.

\section{TESTBED DESIGN}

We refer to our companion paper by Patterson, et.al. ${ }^{4}$ on detail design description of DST. We only provide its brief description here. Fig. 1 is a photo of DST when it is inside the vacuum chamber in JPL's HCIT. As shown in the testbed layout in Fig. 2, DST is configured as Lyot coronagraph based on historical testbed performance in contrast levels. ${ }^{5,6}$ The optical prescription is similar to earlier HLC coronagraph testbeds in HCIT. ${ }^{6,7}$ One distinguishing feature of DST is that it only uses 6 Off Axis Parabolas (OAPs) with no flat fold mirrors to reduce the number of optics in the system, thus, making DST have a very simple optical layout and superior thermal and mechanical stability. ${ }^{4}$

\section{HIGH CONTRAST DEMONSTRATION}

Fig. 3 shows the raw high contrast image achieved after Wavefront Control (WFC) in DST. The averaged raw contrast is $3.82 \times 10^{-10}$ with $10 \%$ broadband light centered at $550 \mathrm{~nm}$ in the 360 degrees dark hole with working 


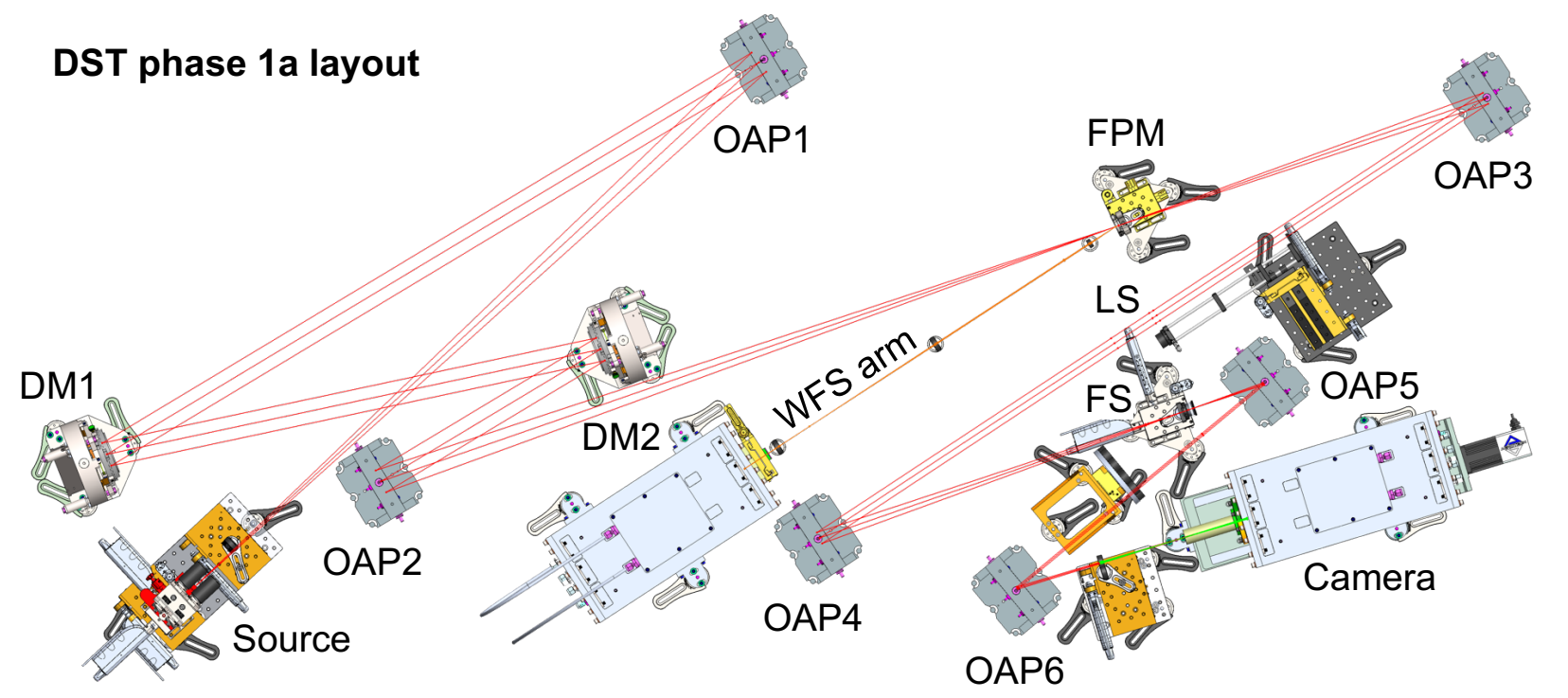

Figure 2. CAD layout of the Decadal Survey Testbed (DST) during commissioning in a Lyot coronagraph architecture. The optical prescription is similar to earlier HLC coronagraph testbeds in HCIT. ${ }^{6,7}$ One distinguishing feature of DST is that it only uses 6 OAPs with no flat fold mirrors to reduce the number of optics in the system, thus, making DST have a very simple optical layout and superior thermal and mechanical stability. ${ }^{4}$
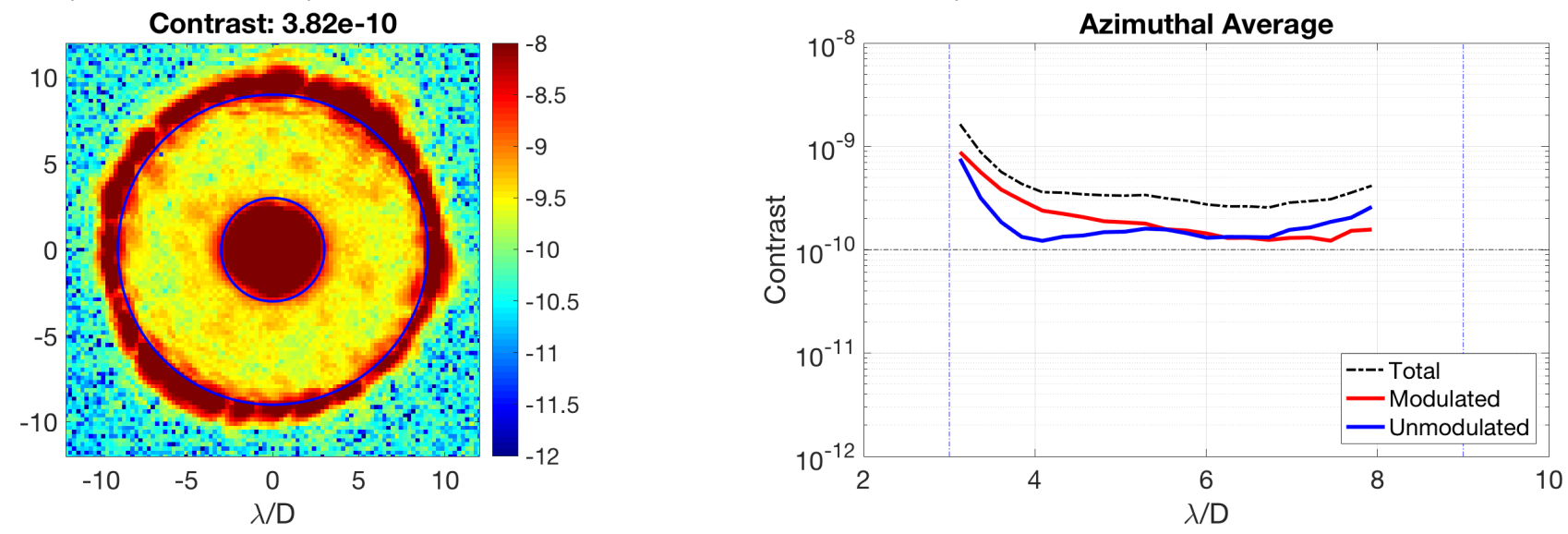

Figure 3. The raw high contrast image achieved in DST and its azimuthal average. The averaged raw contrast is $3.82 \times 10^{-10}$ with $10 \%$ broadband light centered at $550 \mathrm{~nm}$ in the 360 degrees dark hole with working angle between $3 \lambda / D$ and $8 \lambda / D$. See text for more detail.

angle between $3 \lambda / D$ and $8 \lambda / D$. This is the 5 spectral bands' average centered at $528 \mathrm{~nm}, 531 \mathrm{~nm}, 550 \mathrm{~nm}$, $561 \mathrm{~nm}$ and $572 \mathrm{~nm}$ with each band of $11 \mathrm{~nm}$ (or $2 \%$ ) bandwidth respectively. The contrast measurement error is estimated to be less than $5 \%$, which is dominated by a photometry estimation error.

The contrast is measured with linearly polarized light at the source and a linear polarization analyzer in the collimated space before the last OAP (between OAP5 and OAP6 in Fig. 2). As a Focal Plane Mask (FPM), we use a $100 \mathrm{~nm}$ thick simple bare Nickel occulter (on $3 \mathrm{~nm}$ thick titanium layer) with $98 \mathrm{\mu m}$ diameter on the $6.25 \mathrm{~mm}$ thick fused silica (CVI Laser Optics, https://www.cvilaseroptics.com/) substrate with rear surface Anti-Reflection (AR) coated.

The obtained total contrast shown in Fig. 3 can be decomposed and itemized into multiple contributing components as in Table 1. 
When we estimate the normalized complex electric field in the dark hole region, we employ the pairwise estimation scheme ${ }^{8}$ for this electric field estimation process. In this method, "probes" are placed on one of the DMs to modulate the complex electric field across the region of interest. The probes are applied typically in \pm pairs, providing the largest phase diversity and probe simplicity as well as minimizing DM gain uncertainty impact. Given images corresponding to several probe pairs, along with an image with no probes applied at all, we can retrieve the complex electric field at the region of interest. Unlike the phase retrieval algorithm, no mechanical motion is required except DM actuators. We describe our specific use of pairwise estimation process in detail in [7]. In addition to the complex electric field of the residual starlight in the dark hole, our pairwise estimation algorithm ${ }^{7,8}$ also can find a portion of light that does not interact with the applied probes. We refer to this light as "unmodulated" light. As the counterpart to the unmodulated light, we refer to the complex electric field of the residual starlight that responds to the probes as the "modulated" light. We refer [7] for definitions of "unmodulated" and "modulated" lights in more detail.

Accordingly, the inset figures in Fig. 1 shows that the total residual contrast is decomposed into the unmodulated light of $2.01 \times 10^{-10}$ and modulated light of $1.82 \times 10^{-10}$ The black dot, blue, and red curves in the azimuthal average plot of Fig. 3 denote the total, modulated, and unmodulated light, respectively.

We identify two major contributions for the modulated light: the DM actuator Least Significant Bit (LSB) effect and chromatic control residual. On the other hand, the unmodulated light consists of three components: the occulter ghost effect, the testbed Line of Sight (LoS) jitter residual effect, and "unknown" component. In the following section of Sec. 4, we describe each value in more detail.

\section{HIGH CONTRAST ANALYSIS}

Fig. 4 shows the achieved contrasts in various different conditions in earlier testbed configuration where the BK7 material is used instead of fused silica for the FPM substrate. Fig. 4(a), (b) and (c) are the contrast images with $10 \%, 6 \%$ and narrow bandwidth, respectively. For $10 \%$ bandwidth, we use the 5 spectral bands' average centered at $528 \mathrm{~nm}, 539 \mathrm{~nm}, 550 \mathrm{~nm}, 561 \mathrm{~nm}$ and $572 \mathrm{~nm}$ with each band of $11 \mathrm{~nm}$ (or $2 \%$ ) bandwidth respectively. For $6 \%$ bandwidth, we use the 3 spectral bands' average centered at $539 \mathrm{~nm}, 550 \mathrm{~nm}$, and $572 \mathrm{~nm}$ with each band of $11 \mathrm{~nm}$ (or $2 \%$ ) bandwidth respectively. We use the broadband laser system (SuperK Extreme, NKT Photonics) with the tunable spectral filter (SuperK Varia, NKT Photonics) except for the monochromatic

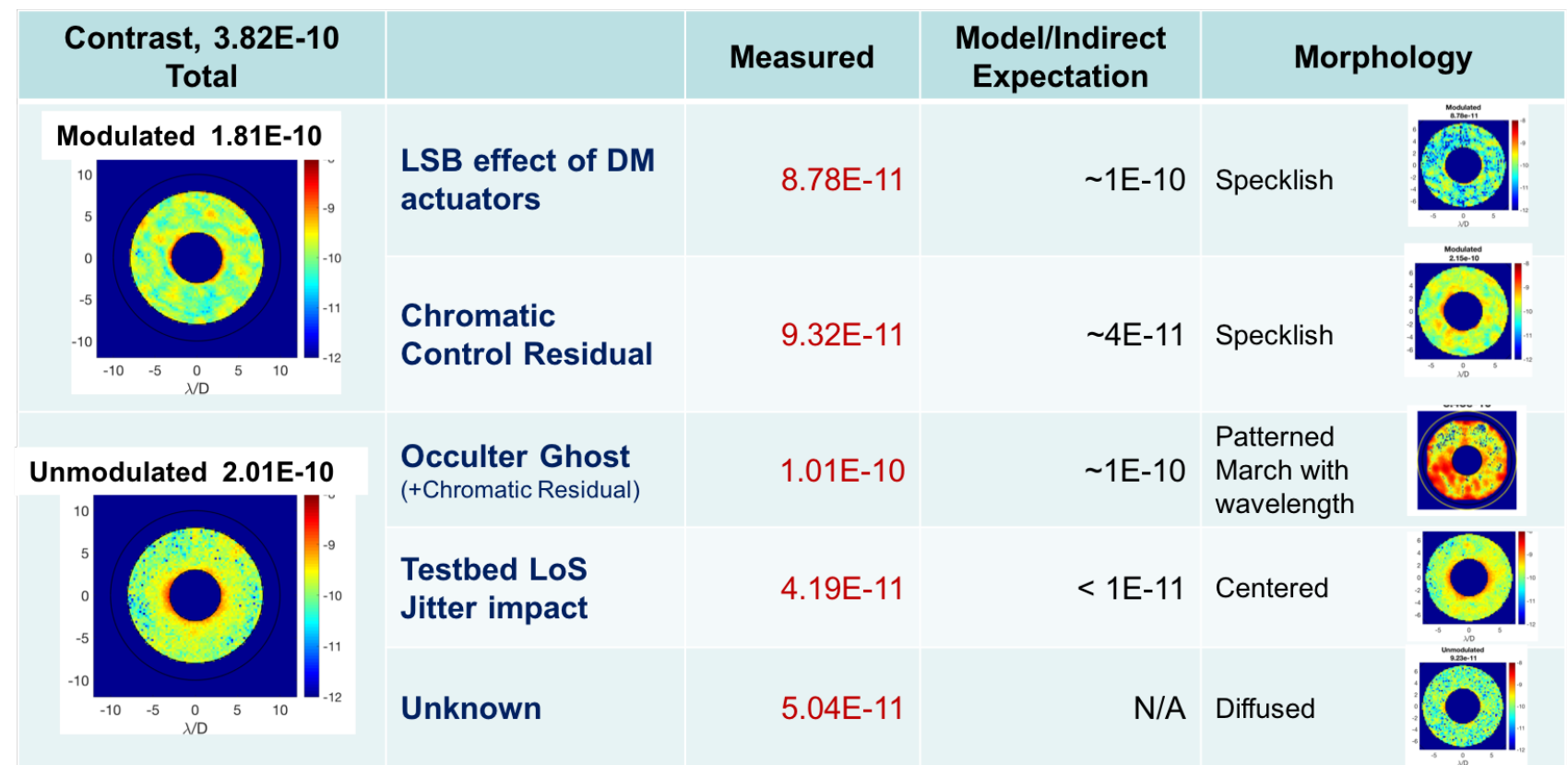

Table 1. The obtained total contrast of $3.82 \times 10^{-10}$ shown in Fig. 3 can be decomposed into multiple contribution components. 


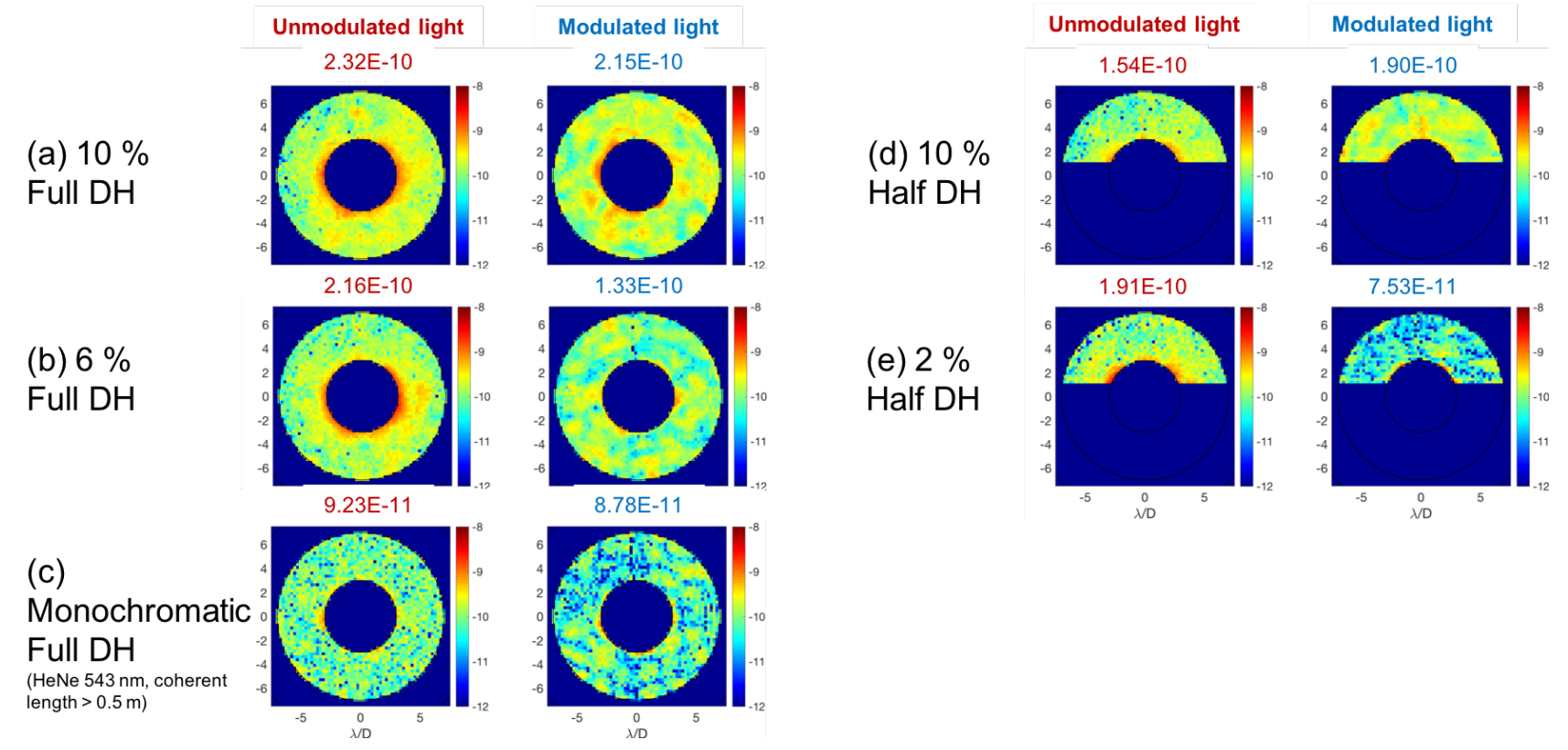

Figure 4. The achieved contrasts in various different conditions in earlier testbed configuration where the BK7 material is used instead of fused silica for the FPM substrate. Based on this result, we can deduce and itemize some of contrast contributions in the measured column of Table 1.

light. For the monochromatic light, we use the HeNe $543 \mathrm{~nm}$ with narrow line width (specified $303 \mathrm{MHz}$ from the manufacturer, REO Inc., model R-33361).

The demonstrated contrast of $3.82 \times 10^{-10}$ in Sec. 3 is broken down into its dominant effects based on these contrast values measured in various conditions. First, we observe the increase of the unmodulated light between (a) and (c) is $1.01 \times 10^{-10}$. We hypothesize this increase is due to two effects. One is the "occulter ghost" effect and the other is "chromatic residual". Since the NKT laser has the shorter coherent length (less than $1 \mathrm{~mm}$ based on its laser linewidth) than the the ghost path length (larger than $12.7 \mathrm{~mm}$ ) while the HeNe has the longer coherent length (approximately $1.0 \mathrm{~m}$ based on its laser linewidth), the occulter ghost is expected to be coherent for (c), incoherent for (a). If coherent, it becomes modulated light. If incoherent, it becomes unmodulated light. Since the pairwise estimation scheme assumes the monochromatic light, any deviation from this assumption will go to the unmodulated light. Therefore, (a) will have more unmodulated light due to larger bandwidth. We name this contribution of unmodulated light "chromatic residual".

The increase of $1.01 \times 10^{-10}$ between (a) and (c) is in good agreement with our independent assessment of the occulter ghost effect of $1.07 \times 10^{-10}$ in Sec. 4.2, implying that the occulter ghost effect is dominant among two effects. Therefore, we assign $1.01 \times 10^{-10}$ as "occulter ghost" measurement in Table 1 .

Second, we find the increase of the modulated light between (a) and (c) is $9.32 \times 10^{-11}$, which is caused by the increased control bandwidth. This is due to the limited capability of the WFC, thus, we name it as "chromatic control residual" measurement in Table 1. The chromatic control residual contribution can be further reduced by improvement of WFC algorithm and calibration accuracy. This value is compared to the theoretical model result of approximately $4 \times 10^{-11}$ which is the DST design limit with ideal calibration (or perfect knowledge) and infinitely small DM actuator LSB.

Third, the smallest modulated light measured at the full dark hole is $8.78 \times 10^{-11}$ in Fig. 4 (c). Since this value is our controllable limit of contrast with the monochromatic light and agrees to the DM LSB effect assessment of $1 \times 10^{-10}$ in Sec. 4.1, we regard this value as the "LSB effect of DM actuators" in Table 1.

Fourth, the smallest unmodulated light measured at the full dark hole is $9.23 \times 10^{-10}$ in Fig. 4(c). We estimate some of this value is from the testbed LoS jitter. Our assessment of the "testbed LoS jitter" is $4.19 \times 10^{-11}$ as 

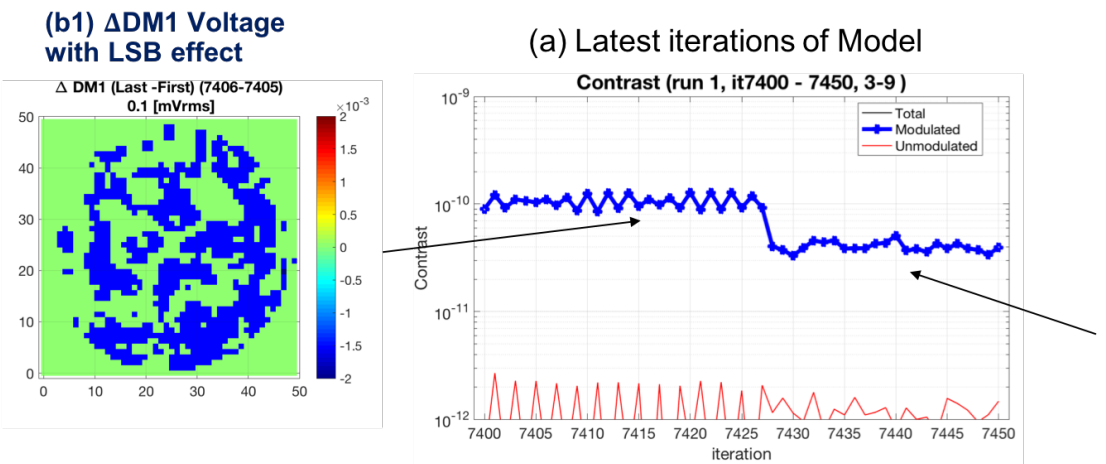

(b2) $\triangle \mathrm{DM} 1$ Voltage with LSB effect + White Voltage Noise

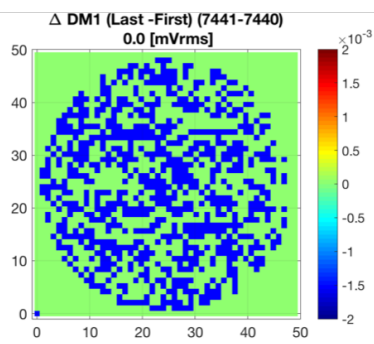

Figure 5. The latest literation of a computer model simulation with the DM actuator LSB effect. We assume the applied voltage is quantized by its DM electronics LSB of 16 bit for $100 \mathrm{~V}$ range. Due to this LSB effect, the contrast is limited by approximately $1 \times 10^{-10}$ as shown earlier part of iteration. See text for description of later iterations.

FALCO Model Result: Contrast vs. LSB

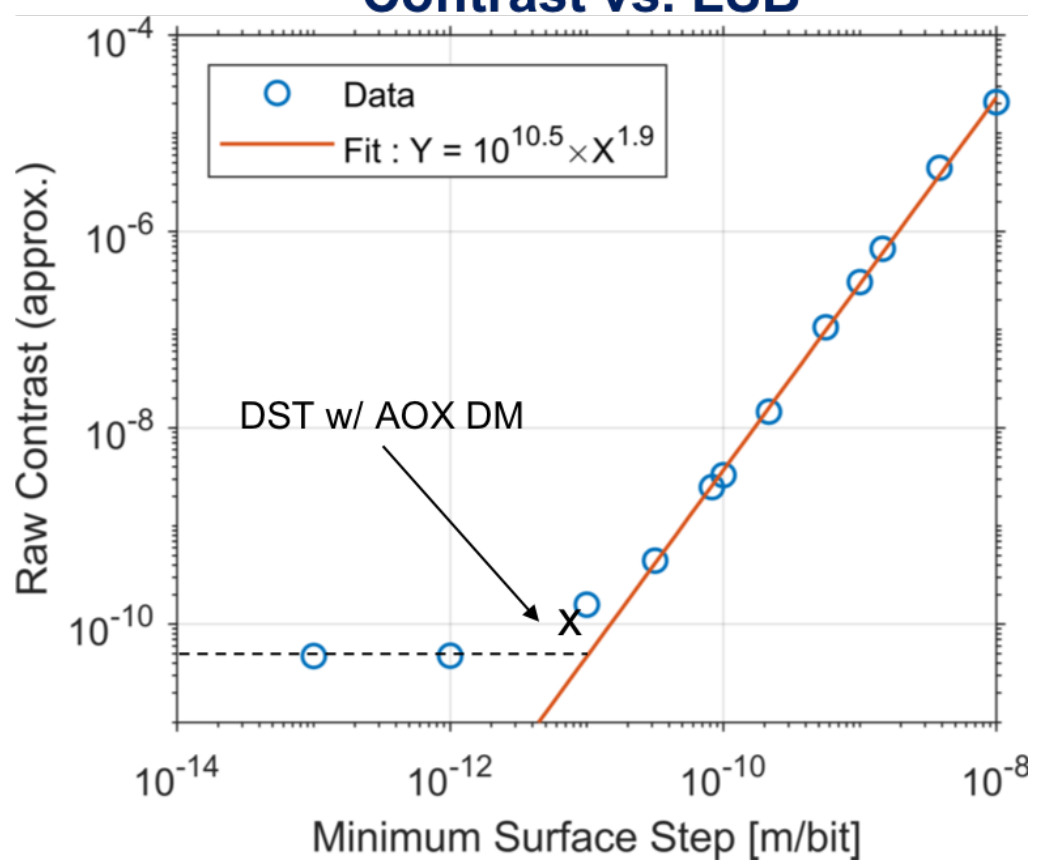

Figure 6. The raw contrast limit vs. the LSB of DM using another independent DST model (FALCO or Fast Linearized Coronagraph Optimizer).

denoted in Table 1 . $^{*}$ The residual of the unmodulated light of $5.04 \times 10^{-11}$ is beyond our understanding as of 2019 and we assign this term as "unknown".

\subsection{LSB effect}

We use compute models to estimate the DM LSB effect. Using on the testbed calibration, we build an in-situ testbed model. Because we use this model to compute the control matrix for the WFC for the testbed, we call this model "control model". ${ }^{6}$

Fig. 5(a) shows the latest WFC iteration result when we run the control model itself as if we run the testbed. In running the control model, we assume the applied voltage is quantized by its DM electronics LSB of 16 bit

${ }^{*}$ DST LoS jitter impact is not described in this paper. 


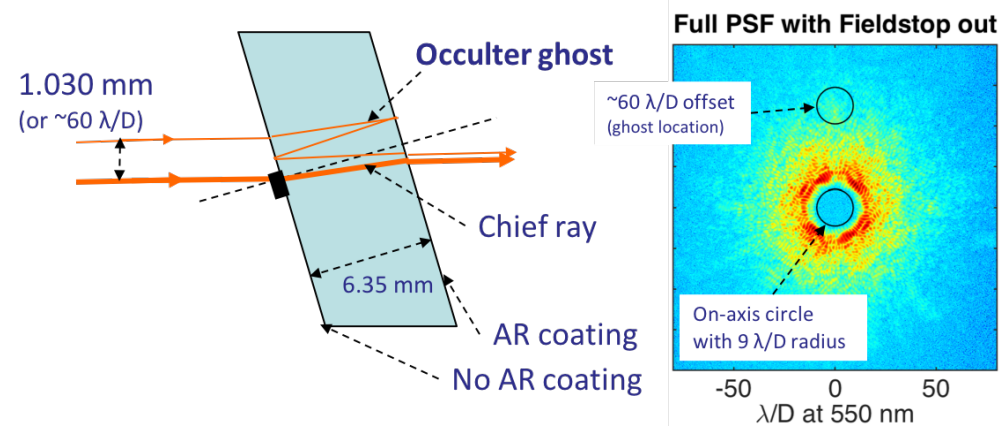

Figure 7. A sketch of DST occulter ghost path when the $6.35 \mathrm{~mm}$ thick fused silica substrate is used with AR coating only on the rear surface.
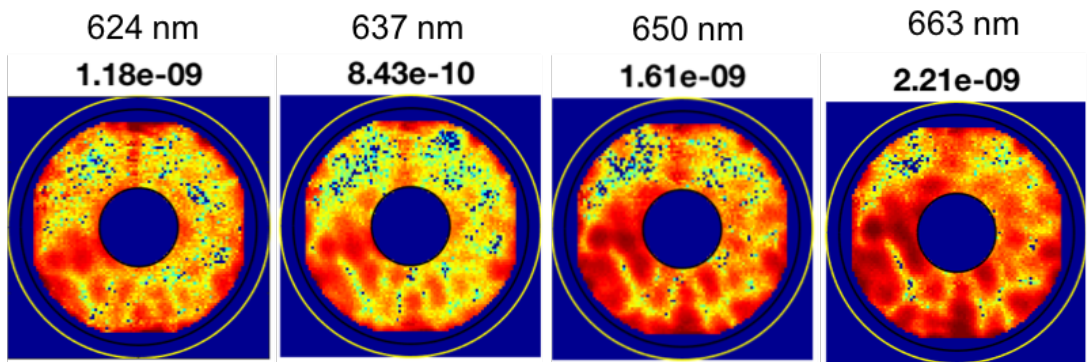

$676 \mathrm{~nm}$

Figure 8. The testbed measurement of the unmodulated light at the higher wavelengths of $624 \mathrm{~nm}, 637 \mathrm{~nm}, 650 \mathrm{~nm}$, $663 \mathrm{~nm}$, and $676 \mathrm{~nm}$. The occulter ghost is dominantly visible and morphologically marching to expected direction as the wavelength increases.

for $100 \mathrm{~V}$ range. Thus, its LSB is $1.5 \mathrm{mV}$ corresponding approximately $6.0 \mathrm{pm}$ surface considering $4 \mathrm{~nm} / \mathrm{V} \mathrm{DM}$ actuator gain. The early (left) part of Fig. 5(a) shows the limit of the DM LSB effect is around $1 \times 10^{-10}$, which is the expectation value for the LSB effect denoted in Table 1. This value also agrees with the smallest modulated light measured at the full dark hole of $8.78 \times 10^{-11}$ in Fig. 4(c).

White voltage noise can be added to the DM voltage to reduce the LSB effect as shown in the later (right) part of Fig. 5(a). However, this technique effect has not been observed in the testbed operation. This reason is not unknown.

In order to understand the DM LSB effect in more systematic way, we run another model by varying the LSB size. Shown in Fig. 6 is the raw contrast limit vs. the LSB of DM using an independent DST model (FALCO or Fast Linearized Coronagraph Optimizer).

As the minimum surface step decreases, the raw contrast obtained approaches its theoretical design contrast limit of approx. $5 \times 10^{-11}$. As the minimum surface step increases, the DM LSB effect becomes dominant and the contrast increases quadratically. DST has the minimum actuator step size of $6 \times 10^{-12} \mathrm{~m} / \mathrm{bit}$. From this curve, the contrast limit due to the LSB effect is approximately $1.00 \times 10^{-10}$, reasonable agreement between two independent models.

\subsection{Occulter ghost effect}

The occulter ghost is caused by imperfect AR coating of the occulter substrate. As sketched in Fig. 7, the occulter ghost effect can be formulated in Eq. (1).

$$
\text { Occulter ghost }=I \cdot R 1 \cdot R 2
$$

, where $I$ is the measurable normalized intensity at around $62 \lambda / D$ (or around $1 \mathrm{~mm}$ ) away from the cheif ray, $R 1$ and $R 2$ are the front and rear reflection coefficients, and the transmission coefficients into/out-of the substrate are ignored. 


\section{BK7 \\ Polarizer \& NO analyzer \\ Unmodulated: $6.02 \mathrm{E}-10(3-8 \mathrm{l} / \mathrm{d})$}

\section{Fused Silica Polarizer \& NO analyzer \\ Unmodulated: $3.30 \mathrm{E}-10(3-8 \mathrm{l} / \mathrm{d})$}
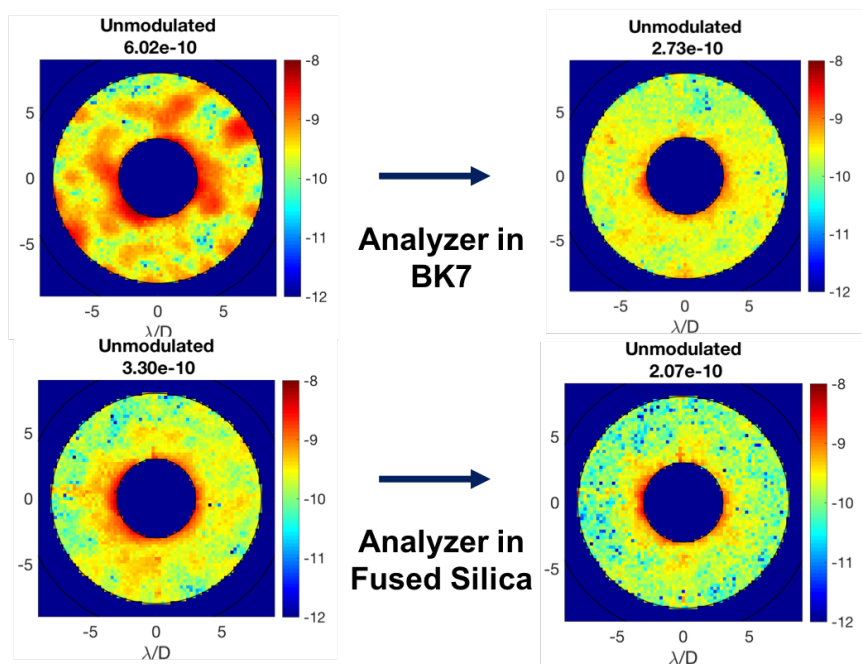

BK7

Polarizer \& Yes analyzer

Unmodulated: $2.73 \mathrm{E}-10$ (3-8 l/d)

\section{Fused Silica}

Polarizer \& Yes analyzer

Unmodulated: 2.07E-10 (3-8I/d)

Figure 9. BK7 vs. fused silica. Two substrate materials are tested with and without the analyzer.

Fig. 8 shows the testbed measurement of the unmodulated light at higher wavelengths of $624 \mathrm{~nm}, 637 \mathrm{~nm}$, $650 \mathrm{~nm}, 663 \mathrm{~nm}$, and $676 \mathrm{~nm}$. As shown in Fig. 8, the occulter ghost is dominantly visible and morphologically marching to the expected direction as the wavelength increases. Using the occulter ghost measurement (unmodulated light) of approximately $1.8 \times 10^{-9}$ at $650 \mathrm{~nm}$ and the measure normalized intensity of $1.80 \times 10^{-6}$ at 52 $\lambda / D$ at $650 \mathrm{~nm}$, the product of the reflection coefficients $R 1 \cdot R 2$ is computed as $1 \times 10^{-3}$ at $650 \mathrm{~nm}$.

According to vendors' AR coating specification, the reflection coefficients are $\times 10$ smaller at $550 \mathrm{~nm}$ comparing to $650 \mathrm{~nm}$. Therefore, we can estimate the occulter ghost effect of $1.07 \times 10^{-10}$ at $550 \mathrm{~nm}$ with the measure normalized intensity of $1.07 \times 10^{-6}$ at $62 \lambda / D$ at $550 \mathrm{~nm}$. This value is in the expectation of the occulter ghost effect in Table 1 and agrees to unmodulated light reduction when we use the long-coherent-length HeNe laser light source as described in earlier in this section.

\subsection{Polarization effect from occulter substrates}

Fig. 9 shows the unmodulated lights with and with polarization control with an analyzer in front of the image plane. The polarization effects are measured with unmodulated light decrease before and after the analyzer is inserted. They are $3.29 \times 10^{-10}$ and $1.23 \times 10^{-10}$, respectively for BK7 and fused silica substrates. This relative ratio agrees to the birefringence measurement result as described in [9].

\section{PLANET DETECTION CAPABILITY}

Future space telescopes with high contrast imaging instruments will make use of post-processing algorithms, which maximize the planet searching and identification capability. To simulate such an observation and to characterize the realistic planet identification capability, we apply a post-processing algorithm to the testbed data as described below.

We first obtain a dark hole of around $4 \times 10^{-10}$ raw contrast after WFC. Then, we continuously take the dark holes with 20 sec exposures, except for two subsequent WFC updates during 15 hours sequence. The 5 wavelength subbands of $528,539,550,561,572 \mathrm{~nm}$ are still used with $11 \mathrm{~nm}(2 \%)$ bandwidth. Fig. 10 shows the " $1 \sigma$ contrast noise" at $5 \lambda / D$ at a function of time. The $1 \sigma$ contrast noise is computed as the standard deviation of contrast values at the all pixels between $5 \lambda / D$ and $5 \lambda / D+1$ pixel. The $1 \sigma$ contrast noise is our measure of the speckle noise when we apply the Angular Differential Imaging (ADI) technique for a post-processing algorithm later.

As shown in Fig. 10, the testbed appears to stabilize over time, i.e., the $1 \sigma$ contrast noise increase rate decreases for later measurement. Whereas in the first sequence takes $3 \mathrm{hr}$ to approach $1 \times 10^{-9}$, the third sequence degrades by same amount in $5 \mathrm{hr}$. Based on our previous experiment, we believe that the contrast noise degradation is largely due to the employed DM (Xinetics $48 x 48$ )'s thermal sensitivity with an additional 


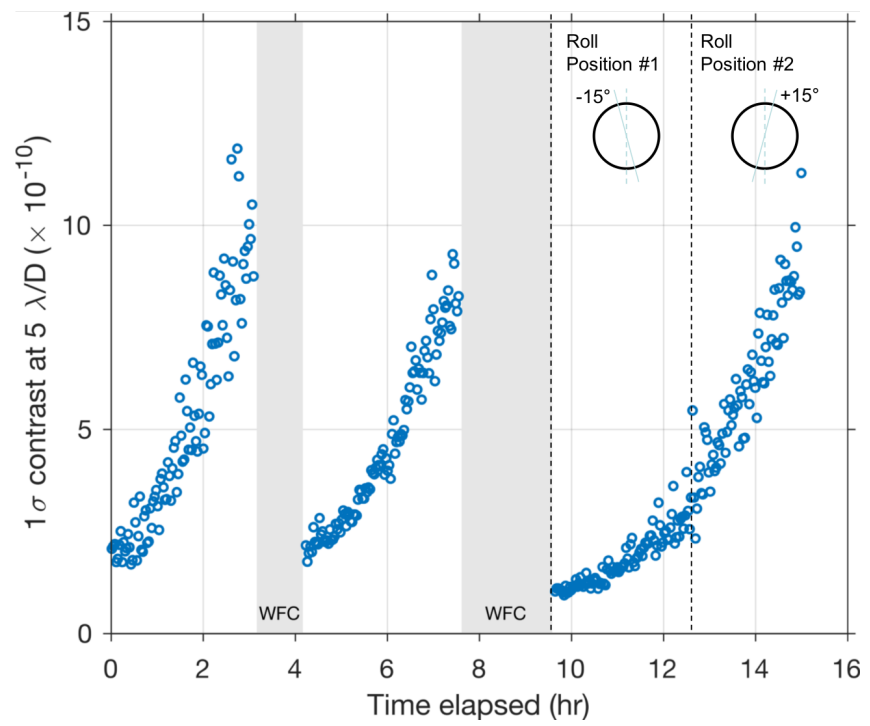

Figure 10. Testbed drift. We continuously take the dark holes with $20 \mathrm{sec}$ exposures, except for two subsequent WFC updates during 15 hours sequence. The plot is the " $1 \sigma$ contrast noise" at $5 \lambda / D$ at a function of time. The $1 \sigma$ contrast noise is computed as the standard deviation of contrast values at the all pixels between $5 \lambda / D$ and $5 \lambda / D+1$ pixel. The $1 \sigma$ contrast noise is our measure of the speckle noise when we apply the Angular Differential Imaging (ADI) technique for a post-processing algorithm in Fig. 11.

(a) Roll position \#1
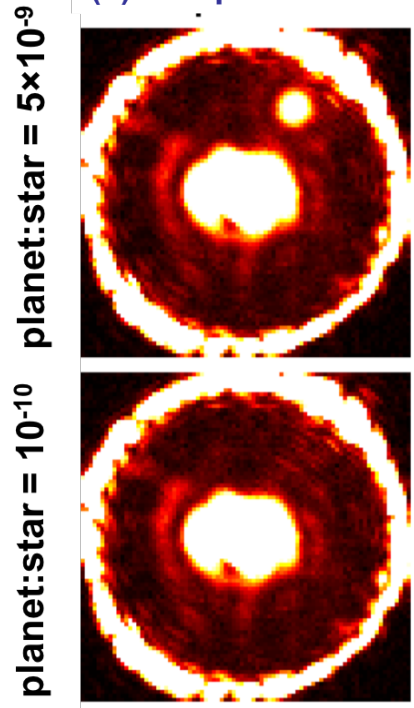

(b) Roll position \#2
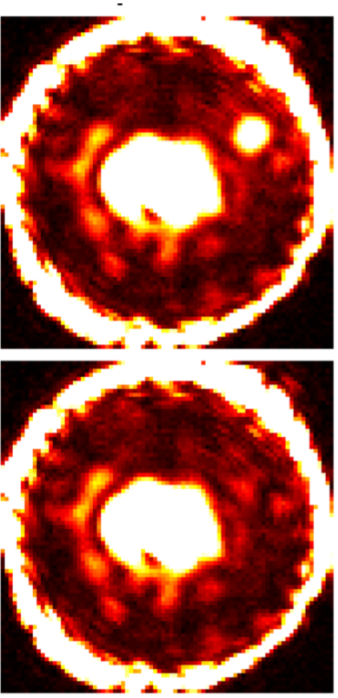

(c) ADI-PCA Residuals
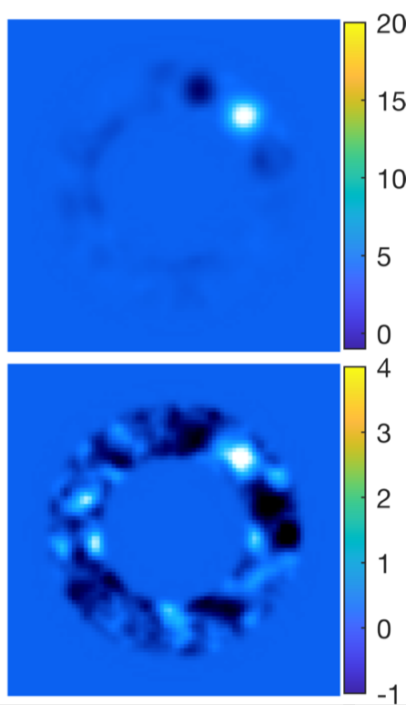

Figure 11. The averaged contrast images at the roll position \#1 (a) and \#2 (b) in Fig. 10. A numerically generated planet is injected for demonstration purpose. We perform the ADI simulation to obtain a differential image shown in (c). The upper and lower parts are same simulation except the planet brightness of $5 \times 10^{-9}$ and $1 \times 10^{-10}$ is considered respectively. This demonstrates that a $1 \times 10^{-10}$ planet imaging is feasible with $4 \sigma$ contrast noise level after ADI is applied in DST.

irregular external disturbance contributing to the earlier drift in the sequence. However, no experiment to prove our hypothesis has been done.

We utilize these testbed data to simulate a simple ADI. We assume a 30 degree roll near middle of the last sequence as indicated in the last sequence of Fig. 10. We also assume a planet at the $5 \lambda / D$ with a certain brightness. In generating a planet, we numerically convolve a point with measured the Point Spread 
Function (PSF). Fig. 11(a) and Fig. 11(b) are the averaged contrast images at the roll position \#1 and \#2 respectively with the numerically generated planet. Then, we perform the ADI simulation using the VIP package ${ }^{\dagger}$ to obtain differential image shown in Fig. 11(c). The upper and lower parts in Fig. 11 are same simulation except the planet brightness of $5 \times 10^{-9}$ and $1 \times 10^{-10}$ is considered respectively.

As a result, the $1 \sigma$ contrast noise floor is measured as $2.5 \times 10^{-11}$ at $5 \lambda / D$. This implies that a $1 \times 10^{-10}$ planet imaging is feasible with $4 \sigma$ contrast noise level as we can see in the bottom of Fig. ??(c) when we numerically inject the $1 \times 10^{-10}$ planet.

\section{CONCLUSION}

We have demonstrated reaching starlight suppression $3.82 \times 10^{-10}$ averaged across the $3 \lambda / D$ and $8 \lambda / D 360$ degree dark hole using the $10 \%$ broadband light at $550 \mathrm{~nm}$. To the best of our knowledge, this raw contrast is the highest $10 \%$ contrast ever achieved in the testbed environment. Furthermore, we could understand the current contrast limit, key contributions and unknowns using testbed measurement and model analysis.

We also find that DST can achieve coronagraph contrast performance capable of detecting a planet as dim as $1 \times 10^{-10}(4 \sigma$ level) at $5 \lambda / D$ after using a standard (or convential) post-processing technique.

For next few years, DST will be utilized to demonstrate various key technological aspects of future coronagraph missions ${ }^{10}$ including different DM technology, low and mid-order Wavefront Sensor (WFS), and segmented pupil obscuration. The result from DST is expected to be valuable and support the future coronagraph missions such as LUVOIR and HabEx.

\section{ACKNOWLEDGMENTS}

This work was carried out at the Jet Propulsion Laboratory, California Institute of Technology, under contract with the National Aeronautics and Space Administration (NASA). We would like to acknowledge funding from NASA's Exoplanet Exploration Program Office, in addition to contributions small and large from the broader WFIRST-CGI, HCIT, and MDL teams.

\section{REFERENCES}

1. The LUVOIR Team, "The Large UV Optical Infrared Surveyor (LUVOIR) final report." https://asd. gsfc.nasa.gov/luvoir/resources/docs/LUVOIR_FinalReport_2019-08-26.pdf, 2019.

2. The HabEx Team, "Habitable Exoplanet Observatory Final Report." https://www.jpl.nasa.gov/habex/ pdf/HabEx-Final-Report-Public-Release.pdf, 2019.

3. W. A. Traub, R. Belikov, O. Guyon, N. J. Kasdin, J. Krist, B. Macintosh, B. Mennesson, D. Savransky, M. Shao, E. Serabyn, and J. Trauger, "Science yield estimation for AFTA coronagraphs," in Society of PhotoOptical Instrumentation Engineers (SPIE) Conference Series, Society of Photo-Optical Instrumentation Engineers (SPIE) Conference Series 9143, p. 0, Aug. 2014.

4. K. Patterson, B.-J. Seo, K. Balasubramanian, T. Chui, B. Crill, J. Gill, B. D. Kern, R. Lam, D. Marx, D. Moody, R. Muller, C. M. Prada, G. Ruane, J. Shaw, F. Shi, N. Siegler, H. Tang, J. Trauger, V. White, D. W. Wilson, K. Yee, and R. Zimmer, "Design description and commissioning performance of a stable coronagraph technology development testbed for direct imaging of Earth-like exoplanets," Proc. SPIE 11117, p. $111171 \mathrm{U}, 2019$.

5. J. T. Trauger and W. A. Traub, "A laboratory demonstration of the capability to image an Earth-like extrasolar planet," Nature 446, pp. 771-773, Apr. 2007.

6. B.-J. Seo, E. Cady, B. Gordon, B. Kern, R. Lam, D. Marx, D. Moody, R. Muller, K. Patterson, I. Poberezhskiy, C. M. Prada, E. Sidick, F. Shi, J. Trauger, and D. Wilson, "Hybrid lyot coronagraph for wfirst: high-contrast broadband testbed demonstration,"

7. B.-J. Seo, B. Gordon, B. Kern, A. Kuhnert, D. Moody, R. Muller, I. Poberezhskiy, J. Trauger, and D. Wilson, "Hybrid Lyot Coronagraph for WFIRST-AFTA: Occulter Fabrication and High Contrast Narrowband Testbed Demonstration," 2015.

\footnotetext{
${ }^{\dagger}$ https://github.com/vortex-exoplanet/VIP
} 
8. A. Give'on, B. D. Kern, and S. Shaklan, "Pair-wise, deformable mirror, image plane-based diversity electric field estimation for high contrast coronagraph," 8151, 2011.

9. K. Balasubramanian, B.-J. Seo, K. Patterson, C. M. Prada, A. J. E. Riggs, H. Zhou, D. Moody, E. Cady, V. White, K. Yee, R. Muller, P. Echternach, F. Greer, and D. Wilson, "Critical characteristics of coronagraph masks influencing high contrast performance," Proc. SPIE 11117-63, 2019.

10. G. Ruane, B. Crill, K. Patterson, C. M. Prada, B.-J. Seo, and N. Siegler, "Decadal survey testbed commissioning roadmap: Demonstrating technology for imaging new worlds." https://exoplanets.nasa.gov/ internal_resources/1170/, 2019. 